Objectives: The study objective was to seek patient input to help characterize ear chondritis An online survey was administered in English or Spanish to participants with self-reported RP.

Methods: Participants were asked questions about their ear pain, including quality, location, duration, aggravating/alleviating factors, timing of onset and duration. Participants were included who reported age $\geq 18$ years, a diagnosis of RP confirmed by a physician, and sufficient symptoms to meet McAdams or Damiani's diagnostic criteria. Participants were categorized as having "typical ear chondritis" if they reported ear pain localized to the pinna with associated redness and swelling. Atypical presentations of ear chondritis were also considered. Results: A total of 685 participants from five continents completed the survey. Among them, 659 met inclusion criteria for subsequent analysis. Most participants were female $(n=574 ; 87 \%)$, white $(n=548 ; 83 \%)$ and from the United States $(n=484 ; 74 \%)$. The median age was 50 years (interquartile range $=41-58)$. In total, $593(90 \%)$ patients reported ear pain, 227 (38\%) had "typical ear chondritis", and $98(16 \%)$ had cauliflower ear.

Ear pain was most commonly described as burning $(n=334,56 \%)$ or throbbing $(n=295,50 \%)$. The most common location of pain was the pinna $(n=373,63 \%)$. Participants reported ear redness $(n=454,60 \%)$ and swelling $(n=405,62 \%)$. Some patients experienced only ear redness without swelling $(n=286,48 \%)$ or only ear swelling without redness $(n=71,12 \%)$. The most common aggravating factors were minor trauma $(n=371,62 \%)$ and stress $(n=358,60 \%)$. The most common alleviating factor was avoidance of touching the ear $(n=374,63 \%)$. Pain was most frequently reported during the daytime $(n=355,60 \%)$ and most likely to occur in either ear at different times $(n=310,52 \%)$. Onset could be gradual $(n=198,33 \%)$ or sudden $(n=155,26 \%)$. Pain typically lasted a few hours $(n=175$, $30 \%$ ) or $2-3$ days $(n=130,22 \%)$. The majority of patients who had pinna pain also had pain in other parts of the ear (e.g. mastoid process, inner ear, whole ear) at some point $(n=394,67 \%)$. In patients with cauliflower ear, the most common location of pain was the pinna $(n=57,58 \%)$ followed by pain inside the ear $(n=53$, $54 \%)$. Most participants reported at least two different types of pain $(n=420$, $64 \%)$.

Conclusion: Ear chondritis in patients with RP has a wide range of clinical presentations and characteristics beyond the typical triad of redness, swelling, and pain localized to the pinna. The description of pain often significantly varies within the same patient. Knowledge of the various distinct characteristics of ear involvement in RP may help physicians recognize and monitor the disease more effectively.

Disclosure of Interests: None declared

DOI: 10.1136/annrheumdis-2021-eular.3440

\section{POS1374 RHEUMATOLOGIC DISEASES AS PRESENTATIONS OF PRIMARY IMMUNODEFICIENCY DISEASES}

S. Kudryashov ${ }^{1}$, L. Karzakova ${ }^{1}$, N. Zhuravleva ${ }^{1}$, I. Sidorov ${ }^{1}$, T. Lutkova ${ }^{1}$. ${ }^{1}$ Federal State Budgetary Educational Institution of Higher Education "The Chuvash State University named after I.N. Ulyanov", Department of Internal Medicine, Cheboksary, Russian Federation

Background: Primary immunodeficiency diseases (PID) occur on average with a frequency of 1: 100000 of the population. In accordance with the classification of PIDS of the International Union of Immunological Societies (IUIS) 9 different groups of innate errors of immunity are distinguished [1]. The polymorphism of the clinical picture, difficulties in recognizing PID cause their late diagnosis and the associated development of irreversible organ damage, complications and high mortality. According to the traditional view, the clinical marker of PID is an infectious syndrome. However, in some cases, PID is detected as a rheumatologic disease.

Objectives: The aim of the study is to analyze the frequency of rheumatologic diseases in adult patients with PID living in one of the subjects of Russia -Chuvashia.

Methods: The material of the study was patient data obtained during a retrospective analysis of 49 outpatient records included in the Republican Register of PID. Diagnosis of various forms of PIDS was carried out in accordance with the criteria developed by the IUIS [1].

Results: During the period from 1993 to January 2020, 49 cases of PID were registered in the adult population of Chuvashia. According to the frequency of PID, common variable immunodeficiency (CVID) is the most common in Chuvashia (26 people). In the second place there is selective IgA deficiency (10 people); in third place there are X-linked agammaglobulinemia (4 people) and hereditary angioedema (4 people). The remaining forms of PID account for 5 cases: 2 cases of Louis-Bar syndrome, 1 case of DiGeorge syndrome, 1 case of Wiskott-Aldrich syndrome and 1 case of Hyper IgE syndrome. The main symptom of PID in $35(71.4 \%)$ patients was heightened susceptibility to infection. In 14 (28.6\%) patients, the clinical picture was dominated by non-infectious presentations (autoimmune, lymphoproliferative and oncological diseases). In 9 patients
(18.3\%), it was manifested by rheumatologic diseases (rheumatoid arthritis, psoriatic arthritis, scleroderma-like syndrome). These symptoms were more characteristic of CVID. With selective IgA deficiency and X-linked agammaglobulinemia rheumatic symptoms were observed only in isolated cases. CVID debuted in 2 cases as rheumatoid arthritis, resulting in the delay in the diagnosis of an average of 5.2 years. Only the detailed immunological and genetic study made it possible to diagnose PID and prescribe adequate treatment - replacement immunoglobulin therapy. This treatment reduced the frequency of infectious manifestations of the disease and it did not significantly improve the course of rheu matological diseases. Therefore, the use of methotrexate and targeted therapy was continued.

Conclusion: In the clinical picture of PID, rheumatic symptoms predominate in $28.6 \%$ of cases, which requires a thorough immunological and genetic examination of patients with rheumatic diseases in order to diagnose PID in a timely manner.

REFERENCES:

[1] Picard C, Bobby Gaspar H, Al-Herz W, et al.J Clin Immunol.2018;38(1):96-128 Disclosure of Interests: None declared

DOI: 10.1136/annrheumdis-2021-eular.3526

\section{POS1375 1 THE EFFECT OF M694V HOMOZYGOSITY ON THE CAROTID INTIMA-MEDIA THICKNESS AND FLOW MEDIATED DILATATION IN PATIENTS WITH FMF RELATED AMYLOIDOSIS}

S. Sahin ${ }^{1}$, M. Romano ${ }^{2}$, F. Guzel ${ }^{3}$, O. Kasapcopur ${ }^{1}$, D. Piskin ${ }^{4}$, I. Yilmaz ${ }^{5}$, E. Demirkaya ${ }^{6} .{ }^{1}$ Istanbul University-Cerrahpasa, Cerrahpasa School of Medicine, Department of Pediatric Rheumatology, Istanbul, Turkey; ${ }^{2}$ Schulich School of Medicine \& Dentistry, University of Western Ontario, Department of Paediatrics, Division of Paediatric Rheumatology, London, Canada; ${ }^{3}$ Gentera Biotechnology, Biotechnology, Istanbul, Turkey; ${ }^{4}$ Schulich School of Medicine \& Dentistry, University of Western Ontario, Department of Epidemiology and Biostatistics, London, Canada; ${ }^{5}$ Epigenetic Health Solutions, Unit of Nephrology, Ankara, Turkey; ${ }^{6}$ Schulich School of Medicine \& Dentistry, University of Western Ontario, Department of Paediatrics, Division of Paediatric Rheumatology and Department of Epidemiology and Biostatistics, London, Canada

Background: There are limited data in FMF associated AA amyloidosis patients regarding the vascular abnormalities including atherosclerosis and endothelial dysfunction, all of which are contributors of cardiovascular disease (CVD) risk And, this risk assessment for future CV events have not yet been evaluated between FMF amyloidosis patients with different genotypes.

Objectives: We aimed to compare early markers of endothelial dysfunction and atherosclerosis, mortality and other disease characteristics in FMF-related amyloidosis patients with a homozygous M694V genotype and in patients with other genotypes with this cross-sectional comparative study.

Methods: For this purpose, patients with FMF-related amyloidosis were assigned according to the MEFV gene mutation to one of the two groups. Group 1: Patients homozygous for M694V (which is the most common genotype associated with the most severe clinical phenotype in FMF) Group 2: Patients homozygous (other than M694V) or compound heterozygous (including M694V) for other MEFV variants. Potential confounders were controlled by excluding the patients with untreated hypertension, diabetes mellitus, obesity, smoking, previous his tory of CVD low glomerular filtration rate (eGFR $<70 \mathrm{~mL} / \mathrm{min}$ ). Flow-mediated dilatation (FMD), pentraxin-3 (PTX3) as early markers of endothelial dysfunction and carotid intima-media thickness (cIMT), fibroblast Growth Factor 23 (FGF23) as an indicator of atherosclerotic vascular disease, all of which are non-invasive tests that are also used to identify subjects at increased risk for future CV events were measured in these 2 groups.

Results: We analyzed demographic, clinical, genetic, survival data and these non-invasive markers of endothelial dysfunction (FMD and PTX3) and atherosclerosis (cIMT and FGF23) in 76 FMF amyloidosis patients with homozygous M694V mutations (Group 1) and 93 FMF amyloidosis patients with two pathogenic mutations apart from M694V homozygosity (Group 2) (Table 1). Brachial artery FMD was significantly lower in Group 1 when compared with Group 2 subjects (6.0 vs. $6.5 \%, p<0.001)$. clMT, FGF23 and PTX3 levels were higher in Group 1 when compared with Group 2 (cIMT: 0.84 vs. $0.67 \mathrm{~mm}, \mathrm{p}<0.001$ FGF23: 52.4 vs. 38.0 pg/dL, $p=0.01$; PTX3: 14.0 vs. $3.2 \mathrm{ng} / \mathrm{mL}, \mathrm{p}<0.001$ ). Time to develop amyloidosis was similar in patients homozygous for M694V (median 10.0 years, $95 \% \mathrm{Cl}: 8.99-11.0$ ) and in Group 2 (median 10.0 years, $95 \% \mathrm{Cl}: 8.97$ 11.0) ( $p>0.05$ ). Mortality rate was significantly higher in Group 1 compared to Group $2(18.4 \%$ vs. $1.1 \%, p<0.001)$ over a median of 7.8 years of follow-up. FMF amyloidosis patients homozygous for M694V had reduced survival (mean survival: 97 months, $95 \% \mathrm{Cl}$ : 94.9 to 101.0 months) as compared to Group 2 (mean survival: 100.7 months, $95 \% \mathrm{Cl}: 100.0$ to 101.3 months) $(p<0.001)$ (Figure 1). The proportion of the clinical manifestations including fever, serositis, erysipelas-like 
erythema, all of which are associated with an FMF attack did not differ between two groups ( $>>0.05)$ apart from arthritis $(76.3 \%$ in Group 1 and $59.1 \%$ in Group 2, $\mathrm{p}<0.05$ ) (Table 1).

Conclusion: In patients with FMF related amyloidosis, M694V homozygosity is associated with lower FMD values and higher cIMT, FGF23 and PTX3 levels. In summary, the data demonstrate that a genotype-phenotype association exists in terms of endothelial dysfunction and atherosclerosis in FMF patients with amyloidosis.

Table 1.

\begin{tabular}{lcccc}
\hline & Group 1 $(\mathbf{n}=\mathbf{7 6})$ & Group 2 $(\mathbf{n = 9 3 )}$ & Total $(\mathbf{n = 1 6 9 )}$ & $\mathbf{p}$ \\
\hline Clinical Findings & $\mathbf{n}(\%)$ & $\mathbf{n}(\%)$ & $\mathbf{n}(\%)$ & \\
\hline Dialysis & $2(2.6)$ & $3(3.2)$ & $5(3.0)$ & $1.00^{*}$ \\
Kidney transplantation & $5(6.6)$ & $4(4.3)$ & $9(5.3)$ & $0.73^{*}$ \\
Kidney biopsy & $68(89.5)$ & $85(91.4)$ & $153(90.5)$ & 0.67 \\
& Median & Median & Median & \\
& & & & \\
Age (years) & $($ Min-max) & (Min-max) & (Min-max) & \\
Age of amyloidosis (years) & $36.5(19.0-49.0)$ & $35.0(22.0-49.0)$ & $36.0(19.0-49.0)$ & 0.16 \\
BMl (kg/m²) & $20.0(13.0-31.0)$ & $20.0(15.0-26.0)$ & $20.0(13.0-31.0)$ & 0.86 \\
HOMA & $26.0(21.0-32.0)$ & $27.0(19.0-32.0)$ & $26.6(29.0-32.0)$ & 0.89 \\
Proteinuria (g/24h) & $2.2(1.2-7.3)$ & $2.4(1.1-8.4)$ & $2.4(1.1-8.4)$ & 0.40 \\
& 7.6 & 4,6 & 5.4 & $<0.001$ \\
\hline
\end{tabular}

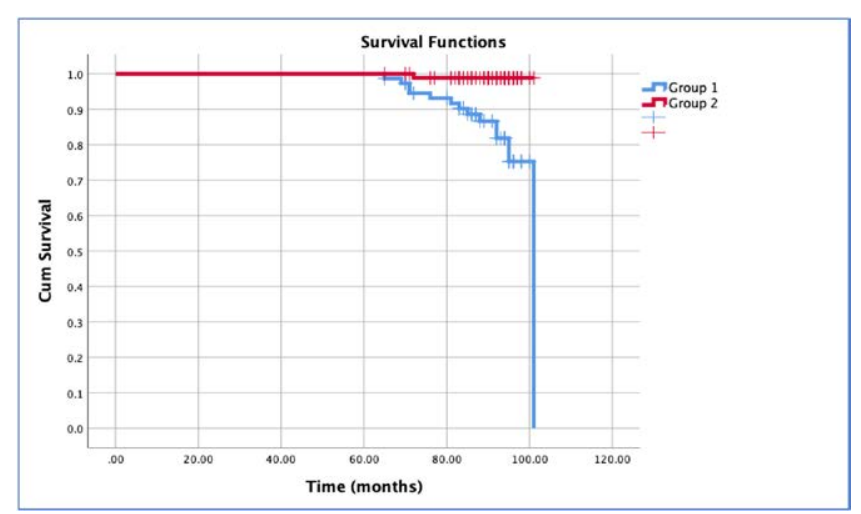

Disclosure of Interests: None declared

DOI: 10.1136/annrheumdis-2021-eular.3582

\section{POS1376 UVEITIS IN 1449 PATIENTS WITH INFLAMMATORY BOWEL DISEASE. STUDY FROM A SINGLE UNIVERSITY CENTER}

L. Sanchez-Bilbao ${ }^{1}$, M. J. García-García ${ }^{2}$, D. Martínez-López ${ }^{1}$, M. RiveroTirado $^{2}$, B. Castro ${ }^{2}$, I. González-Mazón ${ }^{1}$, J. Crespo ${ }^{2}$, M. A. González-Gay ${ }^{1}$, R. Blanco ${ }^{1}{ }^{1}$ Hospital Universitario Marqués de Valdecilla, Rheumatology, Santander, Spain; ${ }^{2}$ Hospital Universitario Marqués de Valdecilla,

Gastroenterology, Santander, Spain

Background: Inflammatory bowel disease (IBD), which includes Crohn's disease (CD), and Ulcerative colitis (UC) are related to Spondyloarthritis (SpA). Ocular manifestations (OM) are well-stablished in axial SpA but not in IBD. It has been classically reported that whereas uveitis with axial $\mathrm{SpA}$ is predominantly anterior, unilateral, acute, and non-recurrent; in IBD it is bilateral, posterior, insidious, and chronic (1).

Objectives: In a large unselected series of IBD, our aim was to assess a) epidemiology and clinical features of uveitis associated to IBD, $\mathbf{b}$ ) to compare patients who developed uveitis and those who did not, and c) its relationship with biological treatment used in IBD.

Methods: Study of all consecutive patients from a single University Hospital during the last 40 years with: a) IBD (CD and UC), and b) uveitis according to Standarization Uveitis Nomenclature (SUN) Working Group. Demographic features, clinical findings, occurrence of other extraintestinal manifestations and treatment were recorded.

Results: We studied 1449 (714 women/735 men) patients with IBD, mean age of $55.2 \pm 15.9$ years.

Uveitis was present in 23 (1.6\%) (38 eyes) of 1448 IBD patients. The most common pattern of uveitis was typically anterior $(n=18 ; 78.3 \%)$, unilateral $(n=19$; $82.6 \%)$, acute $(n=19 ; 82.6 \%)$, and non-recurrent $(n=12 ; 52.2 \%)$.
The comparative study between patients with and without uveitis showed a significant predominance of women (Table 1) in patients with uveitis, as well as erythema nodosum, hidradenitis suppurativa and joint involvement.

Regarding IBD severity, in terms of surgical interventions, and conventional and biological immunosuppressive treatments, there were no significant differences. Conclusion: Although uveitis is more infrequent in IBD than in axial $\mathrm{SpA}$, it is also anterior, unilateral, acute, and non-recurrent in contrast with published data from selected series. Patients with uveitis do not seem to represent more severe phenotype of IBD.

REFERENCES:

[1] Lyons \& Rosenbaum JT. Arch Ophthalmol 1997; 115:61-4.

Table 1. General features of 1448 patients with IBD with and without uveitis.

\begin{tabular}{|c|c|c|c|c|}
\hline & $\begin{array}{l}\text { Overall } \\
(n=1449)\end{array}$ & $\begin{array}{l}\text { Uveitis } \\
(n=23)\end{array}$ & $\begin{array}{l}\text { Non uveitis } \\
(n=1426)\end{array}$ & $\mathbf{p}$ \\
\hline \multicolumn{5}{|l|}{ Main general features } \\
\hline Age, years, mean \pm SD & $55.2 \pm 15.9$ & $49.1 \pm 14.6$ & $55.2 \pm 15.9$ & 0.8 \\
\hline Sex, women/men, $\mathrm{n}$, (\% of women) & $\begin{array}{l}714 / 735 \\
(49.3)\end{array}$ & 17 / 6 (73.9) & $\begin{array}{l}697 / 729 \\
(48.9)\end{array}$ & $0.02^{*}$ \\
\hline $\begin{array}{l}\text { IBD duration, years, mean } \pm \text { SD } \\
\text { IBD Severity }\end{array}$ & $13.2 \pm 9.7$ & $17.4 \pm 10.2$ & $13.1 \pm 8.9$ & 0.08 \\
\hline Surgical Interventions, n (\%) & $289(19.9)$ & $2(8.7)$ & $284(19.9)$ & 0.7 \\
\hline $\begin{array}{l}\text { Conventional Immunosuppressive drugs, } \\
\mathrm{n}(\%)\end{array}$ & $878(60.6)$ & $14(60.9)$ & $863(60.5)$ & 0.5 \\
\hline Biological Therapy, n (\%) & $384(26.5)$ & $7(30.4)$ & $378(26.5))$ & 0.9 \\
\hline TNFi monoclonal antibodies & $384(26.5)$ & $7(30.4)$ & $378(26.5)$ & 0.9 \\
\hline Ustekinumab & $27(1.9)$ & $1(4.3)$ & $27(1.9)$ & 0.5 \\
\hline Other & $23(1.6)$ & $1(4.3)$ & $22(1.6)$ & 0.3 \\
\hline \multicolumn{5}{|l|}{ Extraintestinal manifestations } \\
\hline Cutaneous manifestations, $n$ (\%) (TOTAL) & $125(8.6)$ & $9(39.1)$ & $121(8.7)$ & 0.1 \\
\hline Erythema nodosum, n (\%) & $26(1.8)$ & $6(26.1)$ & $24(1.7)$ & $0.009^{*}$ \\
\hline Pyoderma gangrenosum, $\mathrm{n}(\%)$ & $13(0.9)$ & $1(4.3)$ & $13(0.9)$ & 0.7 \\
\hline Hidradenitis suppurativa, n (\%) & $2(0.1)$ & $1(4.3)$ & $1(0.1)$ & $0.0001^{*}$ \\
\hline Joint involvement, n (\%) (TOTAL) & $131(9)$ & $10(43.5)$ & $121(8.5)$ & $0.0001^{*}$ \\
\hline Axial pattern, n (\%) & $65(4.5)$ & $4(17.4)$ & $58(4.1)$ & $0.0001^{*}$ \\
\hline Peripheral pattern, n (\%) & $64(4.4)$ & $4(17.4)$ & $63(4.4)$ & 0.9 \\
\hline
\end{tabular}

Disclosure of Interests: Lara Sanchez-Bilbao: None declared, María José García-García: None declared, David Martínez-López: None declared, Montserrat Rivero-Tirado: None declared, Beatriz Castro: None declared, Iñigo González-Mazón: None declared, Javier Crespo: None declared, Miguel A González-Gay Speakers bureau: AbbVie, Pfizer, Roche, Sanofi, Celgene and MSD., Grant/research support from: AbbVie, MSD, Jansen and Roche, Ricardo Blanco Speakers bureau: AbbVie, Pfizer, Roche, Bristol-Myers, Janssen, Lilly and MSD., Grant/research support from: AbbVie, MSD and Roche

DOI: 10.1136/annrheumdis-2021-eular.3610

\section{POS1377 SOME PARAMETERS OF ENDOTHELIAL FUNCTION IN PATIENTS WITH IDIOPATHIC AVASCLAR NECROSIS}

K. Gudkevich ${ }^{1}$, N. Martusevitch ${ }^{2}$, E. Dashkevich ${ }^{3} .{ }^{1}$ Minsk City Clinical Hospital No.6, Rheumatology, Minsk, Belarus; ${ }^{2}$ Belarusian State Medical University, Cardiology and Internal Diseases, Minsk, Belarus; ${ }^{3}$ Minsk City Clinical Hospital No.10, Central Hormonal Laboratory, Minsk, Belarus

Background: Avascular necrosis (AN) is a disease, which is the result of blood flow disturbance and necrosis of the bone tissue. It affects predominantly men of working age and steadily progresses to secondary osteoarthritis. AN is a multifactorial disease. In the context of vascular hypothesis it is of particular interest to evaluate homocysteine and its metabolism in patients with AN.

Objectives: The aim of the present study was to evaluate some parameters of endothelial function in patients with $\mathrm{AN}$ of the femoral head or femoral condyles in order to determine possible treatment and prevention options.

Methods: We included 53 patients with AN of the femoral head or femoral condyles into the study. The patients were aged $49(35-62)$ years, 58,49\% $(n=31)$ were male. The inclusion criteria was diagnosis of AN confirmed by X-Ray, CT or MRI. The exclusion criteria were: traumatic AN, Legg-Calve-Pertes disease, connective tissue systemic diseases. We recruited 51 patients into the control group. The groups were comparable by age and gender. In order to analyse engothelial function in the groups we investigated the following serum parameters: homocysteine, vitamin B12, folate. In order to analyse the data obtained we used non-parametric statistics. 